\title{
A Simple Approach to the Synthesis of Hexagonal-Shaped Silver Nanoplates
}

\author{
G. Cynthia Jemima Swarnavalli, ${ }^{1}$ V. Joseph, ${ }^{2}$ V. Kannappan, ${ }^{3}$ and D. Roopsingh ${ }^{3}$ \\ ${ }^{1}$ Department of Chemistry, Women's Christian College, Chennai 600 006, India \\ ${ }^{2}$ Department of Physics, Loyola College, Chennai 600 034, India \\ ${ }^{3}$ Department of Chemistry, Presidency College, Chennai 600 005, India
}

Correspondence should be addressed to V. Joseph, vjoseph62@yahoo.co.in

Received 16 February 2011; Revised 11 May 2011; Accepted 15 June 2011

Academic Editor: Claude Estournes

Copyright (C) 2011 G. Cynthia Jemima Swarnavalli et al. This is an open access article distributed under the Creative Commons Attribution License, which permits unrestricted use, distribution, and reproduction in any medium, provided the original work is properly cited.

This paper deals with the synthesis of hexagonal-shaped silver nanoplates with diameters ranging from 15 to $20 \mathrm{~nm}$ along with a smooth nanobulk of $120 \mathrm{~nm}$. These nanoplates were prepared by a kinetically controlled solution growth method using mild reducing agent dextrose, polyvinylpyrrolidone (PVP $M_{\mathrm{w}} 40 \mathrm{kDa}$ ) as the capping agent and urea as a habit modifier and at a moderate temperature of $50^{\circ} \mathrm{C}$. The crystal structure of the highly faceted particles consists mostly of (111) surfaces as revealed by both X-ray diffraction (XRD) studies and transmission electron microscopy (TEM).

\section{Introduction}

Silver (Ag) nanoparticles of different shapes possess unique and tunable optical properties. Their surface Plasmon resonance (SPR) and surface-enhanced Raman spectroscopy (SERS) can be used for optical labels, contrast enhancement agents, near-field optical probes, and chemical and biological sensors [1-6]. They can be treated as fluorescent analogue tracer in biological assay [7]. These properties and applications of silver nanoparticles relate to their morphology and size. Hence, controlled synthesis of silver nanoparticles with well-defined shapes and sizes is pursued for investigating their size and morphology-dependent properties and their practical applications. A number of solution-phase methodologies have been developed to synthesize the nanoparticles successfully with a wide range of well-defined shapes $[8,9]$ such as cubes and octahedrons [10, 11], tetrahedrons [12], icosahedrons and decahedrons [13], wires/rods [14, 15], plates with varying profiles such as triangular plates [16], and nanodiscs [17]. Among these particles, two-dimensional nanoplates have particularly attracted attention due to their ability to control optical properties. They exhibit intense inplane dipole surface Plasmon resonance peak which is tunable in Visible and NIR regions by simply controlling its edge length to thickness ratio and/or truncation degree of tips which is not possible with spherical Ag particles. Strong absorption coefficient of Ag nanoplates over the broad UVNIR spectral regions and the scattering of light by these nanoplates make them an ideal class of light trapping material which enhances charge carrier generation in organic photovoltaic films [18]. Nanoplates are generally synthesized by transformation of spherical nanoparticles into plates by photo-induced methods. Mirkin's [19] group pioneered this strategy by using fluorescent light and laser with controlled wavelength. Visible light [20], UV light [21], microwaves [22] were also used to induce the conversion of spheres to nanoplates. Later on, kinetically controlled reduction of $\mathrm{Ag}^{+}$ ions provided a simple cost effective method to synthesize plate like morphologies [23]. Seed-mediated growth method $[24,25]$ is another method used for the synthesis of nanoplates. Xionghui and Aixia [26] have used sulphuric acid as a modifier to prepare hexagonal silver nanoplates. All of these methods require high temperature and long duration of reaction time. In the present work, Ag nanoplates were prepared by heterogeneous nucleation method using preformed silver seeds of size $3-10 \mathrm{~nm}$. The growth solution contained reagents according to a method proposed by $\mathrm{Lu}$ and Chou [27]. The reaction conditions and the molecular 
weight of the capping agent are altered to follow a kinetically controlled route to synthesize hexagonal plates. Moderate temperature of $50^{\circ} \mathrm{C}$ was used to synthesize hexagonal plates with a reaction time of 3 hrs. Urea was added to the growth solution as a habit modifier. Urea is known to change crystal habits in bulk crystals [28]. PVP $\left(M_{\mathrm{w}} 40 \mathrm{kDa}\right)$ was used as a capping agent. Silver nanoparticles, $3-10 \mathrm{~nm}$ in diameter, were used as seeds to grow hexagonal silver nanoplates of 15$20 \mathrm{~nm}$ size.

\section{Materials and Methods}

2.1. Materials. Qualigen samples of silver nitrate $\mathrm{AgNO}_{3}$ and polyvinylpyrrolidone (PVP, $M_{\mathrm{w}} 40 \mathrm{k} \mathrm{Da}$ ) and Merck samples of dextrose, urea, and trisodium citrate were used as such. All solutions were prepared in deionized water.

2.2. Synthesis of Silver Seeds. $20 \mathrm{~mL}$ aqueous solution of silver nitrate $(0.156 \mathrm{M})$ was heated to boiling, $2 \mathrm{~mL}$ of sodium citrate $(0.025 \mathrm{M})$ solution was added to it, and the solution was stirred for 3 minutes and was allowed to react for 5 minutes. The solution turned yellow indicating the formation of silver nanoparticles of size 3-10 $\mathrm{nm}$.

2.3. Preparation of Growth Solution. A growth solution containing $20 \mathrm{~mL}$ of aqueous solution of $\mathrm{AgNO}_{3}(0.156 \mathrm{M})$ and $20 \mathrm{~mL}$ of urea solution $(0.63 \mathrm{M})$ was prepared in a $100 \mathrm{~mL}$ conical flask. To this flask was added $20 \mathrm{~mL}$ alkaline solution of PVP $\left(1 \mathrm{~g} / 1 \mathrm{~g}\right.$ of $\left.\mathrm{AgNO}_{3}\right)$. This solution was used as the growth solution.

2.4. Kinetically Controlled Synthesis of Silver Nanoplates. Silver nanoplates with hexagonal shape were prepared by adding $20 \mathrm{~mL}$ of seed solution to the growth solution in a flask and mixed well by stirring. $20 \mathrm{~mL}$ of an ice cold aqueous solution of dextrose $(0.313 \mathrm{M})$ was added to the flask dropwise with continuous stirring at room temperature. The solution turned jet black and was heated in a thermostat for $3 \mathrm{hrs}$ at $50^{\circ} \mathrm{C}$. The silver nanoparticles were centrifuged at $4000 \mathrm{rpm}$ to produce the precipitate and analyzed by UVVisible absorption spectra, XRD, and TEM.

2.5. Characterization. UV-Visible spectrum of the silver nanoparticles was recorded using a Cary $5 \mathrm{E}$ UV-VIS-NIR spectrophotometer. Powder X-ray diffraction (XRD) patterns were measured using (RICHSEIFER) powder diffractometer, using nickel filtered copper $K$-alpha radiations $(\lambda=$ $1.5461 \AA$ ) with a scanning rate of $0.02^{\circ}$. The diffraction pattern was recorded in the range of $5-70^{\circ}$. The HRTEM images of the silver nanoparticles were recorded using a JEOL JEM 3010 instrument with a UHR pole piece electron microscope operating at $200 \mathrm{kV}$.

\section{Results and Discussion}

3.1. Absorption Spectra. The UV-Vis absorption spectra of freshly prepared samples are given in Figure 1. The seed solution shows an intense band centered at $416 \mathrm{~nm}$, and the

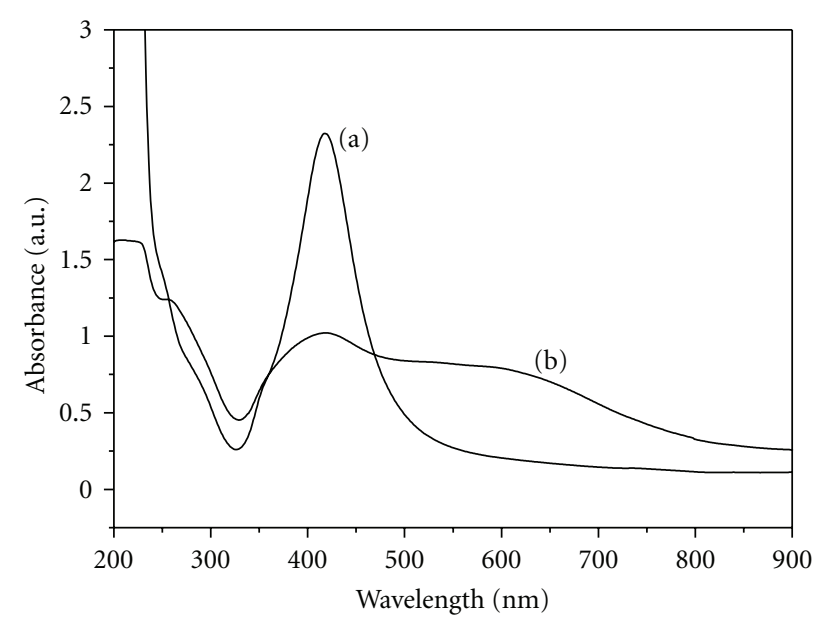

Figure 1: UV-Visible spectra of (a) silver seeds and (b) product solution containing hexagonal silver nanoplates.

particle size is around $3-10 \mathrm{~nm}$. For small nanoparticles, the peaks in the absorption spectra are due to light absorption only. The product solution containing the hexagonal nanoplates shows two bands and a strong SPR absorption peak at about $418 \mathrm{~nm}$ and a shoulder at about $586 \mathrm{~nm}$ are contrary to a single SPR band in the range of 390 and $430 \mathrm{~nm}$ for spherical Ag nanoparticles. Additional peak at $586 \mathrm{~nm}$ is attributed to reduction in symmetry, and the number of peaks correlates with the number of ways the electron density can be polarized [29]. The peak at $416-418 \mathrm{~nm}$ is attributed to the transverse oscillation of electrons and has contributions from the light scattering as well. The new SPR band at $586 \mathrm{~nm}$ has originated from the hexagonal-shaped plates. This peak is attributed to longitudinal oscillation of electrons which can be shifted even up to $1000 \mathrm{~nm}$ in the near-IR region when the aspect ratio of the nanoplates increases [30]. The UV-Visible SPR information is an evidence for the generation of the Ag nanoplates with hexagonal shape.

3.2. X-Ray Diffraction Studies. The crystalline character of the silver nanoplates is supported by XRD technique. Appearance of the XRD peaks (Figures 2(a) and 2(b)) corresponding to seed and product particles demonstrates typical nature of pure FCC crystalline silver (JCPDS Card no. 893722).

A comparison among (111), (200), and (220) diffraction intensities reveals that the ratio between the (111) and (200) peaks is much lower than that of the conventional bulk silver. This fact indicates that the obtained silver nanoplates are dominantly ruled by (111) planes. The diffraction peak for the (220) lattice planes is also present, but its intensity is very weak. Interestingly, there is an obvious increase in the diffraction intensity of the (111) peak relative to that of the (200) peak in going from particles in seed solution to product solution (Figure 2(b)). This is a strong evidence of the preferential growth of the particles along (111) directions. The formation of nanoplates with essentially (111) facets may be the result of the lower free energy of the (111) planes 


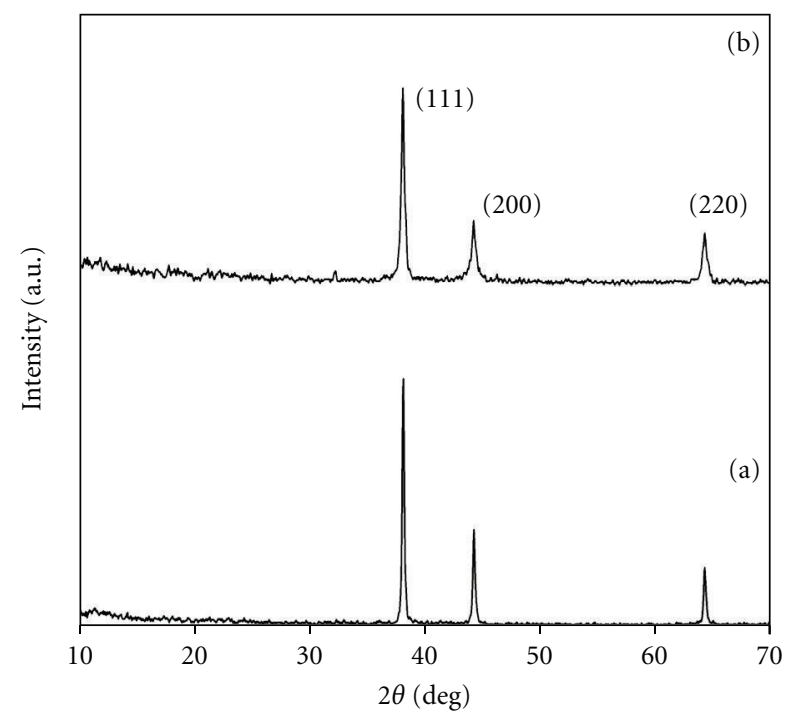

Figure 2: Typical X-ray diffraction patterns of (a) Ag nanoseeds and (b) product solution containing hexagonal silver nanoplates.

and the effective adsorption of urea and PVP to the (100) plane.

3.3. Transmission Electron Microscopy. The particle structure and size distribution can be determined by transmission electron microscopy (TEM). The electron micrographs of PVP-stabilized silver nanoparticles in the seed solution and product solution are given in Figures $3(\mathrm{a})$ and $3(\mathrm{~b})$. It may be seen that in the seed solution (Figure 3(a)) the particles are spherical and moderately monodispersed. In the product solution (Figure 3(b)), however, the images reveal well-defined hexagonal facets present in many of the nanoparticles. A twin boundary in the middle of the particles can be seen when viewing these nanoparticles at different angles (e.g., Figure 3(b) particles 1, 2, 3). We can also see a nanobulk with a smooth surface of about $120 \mathrm{~nm}$ in size with a highly faceted hexagonal shape (Figure 3(c)). (The diameter of the hexagonal particles is determined as the mean of the three distances between opposite corners.) The longest side has a length of around $100 \mathrm{~nm}$ and the shortest side $60 \mathrm{~nm}$. It may be the twinning boundary that allows the $\mathrm{Ag}$ nanoplates to grow into microplates. The bulk may also be a single crystal according to the "surfacewrapping" mechanism proposed by Feng and Zeng [31]. To further understand the crystal habit of the hexagonal silver nanoplates prepared in this study, a high-resolution TEM characterization was performed on the hexagonal silver nanoplates. HRTEM image of a hexagonal-shaped silver nanoplate (Figure 3(d)) shows the lattice planes of the crystal. The plate seems to contain twin boundaries, which is typical for many of the nanoparticles obtained in this study. The lattice planes have a $d$-spacing of $2.36 \AA$ for adjacent lattice planes that corresponds to the (111) planes of face-centered cubic silver. In addition to the presence of twin boundaries, the particle contains (111) and (200) lattice planes with the (111) planes covering a much larger fraction of the particle surface.
3.4. Mechanism of Formation. The mechanism of kinetically controlled synthesis of the hexagonal Ag nanoplates can be discussed as follows. In order to get plate like structure, the precursor reduction rate must be greatly reduced, forcing the $\mathrm{Ag}$ atoms to form seeds through random hexagonal close packing with the inclusion of stacking faults. This is achieved by using wider growth conditions. Firstly a mild reducing agent dextrose was used which promotes the formation of twinned seeds in addition to making the synthesis a kinetically controlled one. Xiong and Xia have [32] demonstrated that the use of a mild reducing agent such as citric acid or ascorbic acid can promote the formation of twined seeds. Murphy and Jana [33] have shown that the addition of preformed seeds to a metal precursor in the presence of a weak reducing agent effectively isolates the nucleation and growth events allowing control over the size and shape of the resulting nanoparticles. Secondary nucleation during the growth stage was inhibited. Secondly, urea a habit modifier PVP as capping agent, helps in the formation of twodimensional plate-like structures. Use of PVP in anisotropic synthesis of silver nanoplates and preferential adsorption of PVP to the (100) plane has been reported in literature $[9,34]$. Thus (100) plane is retained, and preferential addition of silver atoms to the (111) planes was facilitated. In addition to being a habit modifier by getting adsorbed to specific crystal planes, urea was reported [27] to change the reaction path significantly by being a source of cyanate and carbonate ions and thus forming the composite intermediates $\mathrm{AgOCN}$ and $\mathrm{Ag}_{2} \mathrm{CO}_{3}$.

Decomposition of urea in solution occurs as follows [27]

$$
\mathrm{NH}_{2} \mathrm{CO} \mathrm{NH} \mathrm{NH}_{2} \longrightarrow \mathrm{NH}_{4}{ }^{+}+\mathrm{OCN}^{-}
$$

In an alkaline solution, cyanate ions react with hydroxide ions to form carbonate ions and ammonium ion according to

$$
\mathrm{OCN}^{--}+\mathrm{OH}^{-}+\mathrm{H}_{2} \mathrm{O} \longrightarrow \mathrm{CO}_{3}{ }^{2-}+\mathrm{NH}_{4}{ }^{+}
$$

The intermediates were then reduced by dextrose to silver at a relatively slow rate. The kinetically controlled reaction pathway provided sufficient time for the PVP molecules and urea to adsorb onto the silver surfaces. As a result, we need not add many PVP molecules to obtain a uniform dispersion of nanosilver. Some of the urea might have chelated with silver, further slowing down the reduction reaction of the silver ion, and significantly altered the reduction kinetics. The shape of silver nanoparticles is determined by the nature of reducing agent, capping agent, molar concentration ratio of capping agent to silver nitrate, temperature, and reaction time. In the case of mild reducing agent like dextrose, the rate of reduction of $\mathrm{Ag}^{+}$is moderate, and, when $\mathrm{PVP} / \mathrm{AgNO}_{3}$ ratio is $1: 1, \mathrm{PVP}$ can act mostly as capping agent and may not be a reducing agent in the present study. Yang et al. [35] have reported the solvothermal synthesis of hexagonal $\mathrm{Ag}$ nanoplates in DMF assisted by PVP at $140^{\circ} \mathrm{C}$. The reaction time was 6 hrs. He has proposed that as $\mathrm{PVP} / \mathrm{AgNO}_{3}$ ratio decreases, anisotropy increases, but, in the absence of PVP only spherical particles were obtained. In the present study, the temperature is considerably less $\left(50^{\circ} \mathrm{C}\right)$, and the duration 


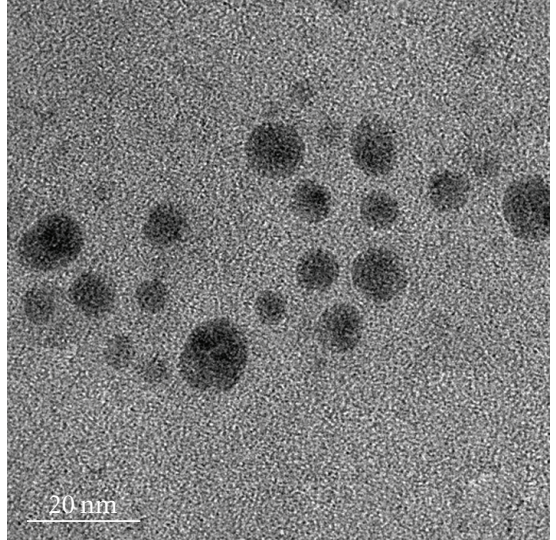

(a)

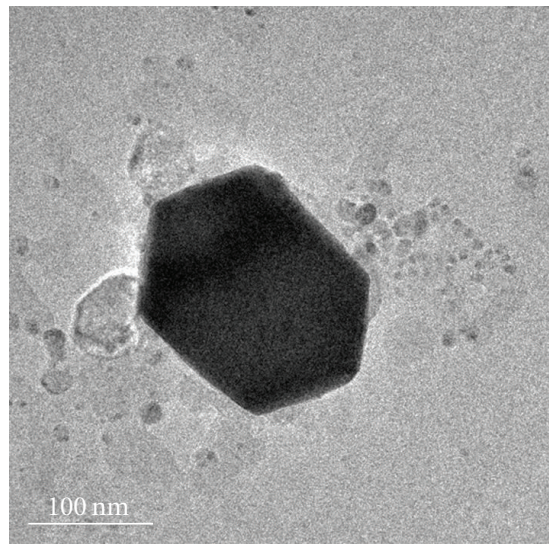

(c)

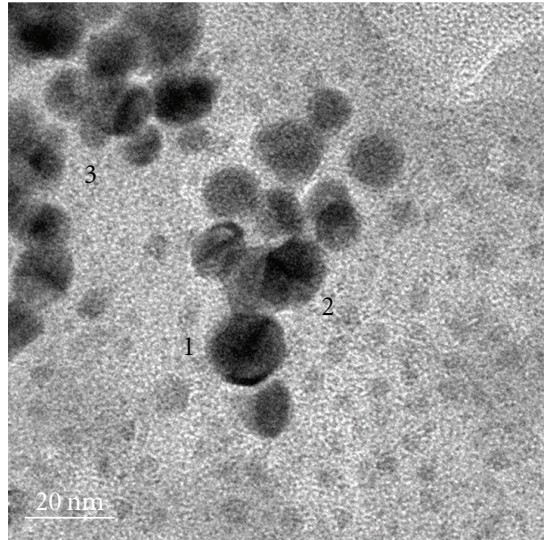

(b)

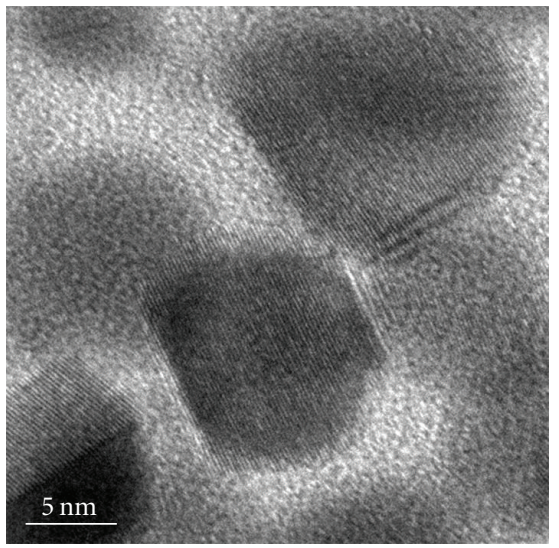

(d)

Figure 3: (a) TEM image showing the spherical Ag nanoparticles of the seed solution (3-10 nm scale bar 20 nm), (b) faceted hexagonal Ag nanoparticles of 15-20 nm with twin boundaries (scale bar $20 \mathrm{~nm}$ ), (c) highly faceted hexagonal Ag nanobulk with $120 \mathrm{~nm}$ size (100 nm), (d) highly faceted hexagonal Ag nanoplates with twin boundary showing a (111) plane with a (d) spacing of $2.36 \AA$.

of reaction is $3 \mathrm{hrs}$. These conditions favour anisotropy, and the PVP gets adsorbed preferentially to (100) facets of the FCC silver, and this increases the growth rate along (111). All these factors favored the formation of hexagonal nanoplates of size $15-20 \mathrm{~nm}$.

\section{Conclusion}

The use of a mild reducing agent like dextrose, higher concentration of $\mathrm{AgNO}_{3}$, polyvinylpyrrolidone (PVP), and urea as a modifier maneuvered the reduction of $\mathrm{AgNO}_{3}$ in a kinetically controlled pathway to produce hexagonal silver nanoplates at a moderate temperature of $50^{\circ} \mathrm{C}$. The reaction time is also considerably less when compared to other existing methods. Moderately monodispersed hexagonal plates of size $15-20 \mathrm{~nm}$ were achieved by the combined use of urea and PVP by adsorption of both to specific crystal planes. The crystal structure was confirmed by XRD data and was determined to possess mostly (111) facets and smaller regions of (100) facets as particle size increases. The formation of these hexagonal nanoplates is attributed to the possible intermediate formation to slow down the synthesis and adsorption of urea and PVP to specific crystal planes. The present work may help to modify optical properties of $\mathrm{Ag}$ nanoplates in the visible and near infrared (NIR) region by controlling their edge length to thickness ratio. These properties and applications of silver nanoparticles relate to their morphology and size.

\section{Acknowledgment}

One of the authors (G. C. J. Swarnavalli) is grateful to University Grants Commission (UGC), New Delhi, for the awards of Teacher Fellowship under the Faculty Development Programme (FDP). The authors thank SAIF and Nano facilities at IIT Madras (a unit of DST) for recording UVVisible spectra and TEM micrographs. The authors thank Dr. S. Dinakaran, Department of Physics, Prathyusha Institute of Technology and Management (PITAM), Aranvoilkuppam, for his help and fruitful discussions.

\section{References}

[1] Y. Sun and A. N. Changhua, "Review article, shaped gold and silver nanoparticles," Frontiers of Materials Science, vol. 5, no. 2, pp. 1-24, 2010. 
[2] Y. Yang, S. Matsubara, L. Xiong, T. Hayakawa, and M. Nogami, "Solvothermal synthesis of multiple shapes of silver nanoparticles and their SERS properties," Journal of Physical Chemistry C, vol. 111, no. 26, pp. 9095-9104, 2007.

[3] Y. Xiong, J. M. McLellan, J. Chen, Y. Yin, Z. Y. Li, and Y. Xia, "Kinetically controlled synthesis of triangular and hexagonal nanoplates and their SPR/SERS properties," Journal of the American Chemical Society, vol. 127, no. 48, pp. 17118-17127, 2005.

[4] C. J. Murphy, T. K. Sau, A. M. Gole et al., "Anisotropic metal nanoparticles: synthesis, assembly, and optical applications," Journal of Physical Chemistry B, vol. 109, no. 29, pp. 1385713870, 2005.

[5] A. R. Tao, S. Habas, and P. Yang, "Shape control of colloidal metal nanocrystals," Small, vol. 4, no. 3, pp. 310-325, 2008.

[6] C. M. Cobley, S. E. Skrabalak, D. J. Campbell, and Y. Xia, "Shape-controlled synthesis of silver nanoparticles for plasmonic and sensing applications," Plasmonics, vol. 4, no. 2, pp. 171-179, 2009.

[7] J. Yguerabide and E. E. Yguerabide, "Light-scattering submicroscopic particles as highly fluorescent analogs and their use as tracer labels in clinical and biological applications," Analytical Biochemistry, vol. 262, no. 2, pp. 137-156, 1998.

[8] Y. Sun and Y. Xia, "Shape-controlled synthesis of gold and silver nanoparticles," Science, vol. 298, no. 5601, pp. 2176 2179, 2002.

[9] J. Zeng, Y. Zheng, M. Rycenga et al., "Controlling the shapes of silver nanocrystals with different capping agents," Journal of the American Chemical Society, vol. 132, no. 25, pp. 8552-8553, 2010.

[10] J. J. Zhu, C. X. Kan, X. G. G. Zhu et al., "Synthesis of perfect silver nanocubes by a simple polyol process," Journal of $\mathrm{Ma}$ terials Research, vol. 22, no. 6, pp. 1479-1485, 2007.

[11] F. R. Fan, DE. Y. Liu, Y. F. Wu et al., "Epitaxial growth of heterogeneous metal nanocrystals: from gold nano-octahedra to palladium and silver nanocubes," Journal of the American Chemical Society, vol. 130, no. 22, pp. 6949-6951, 2008.

[12] J. Zhou, J. An, B. Tang et al., "Growth of tetrahedral silver nanocrystals in aqueous solution and their SERS enhancement," Langmuir, vol. 24, no. 18, pp. 10407-10413, 2008.

[13] M. Tsuji, M. Ogino, R. Matsuo et al., "Stepwise growth of decahedral and icosahedral silver nanocrystals in DMF," Crystal Growth and Design, vol. 10, no. 1, pp. 296-301, 2010.

[14] P. S. Mdluli and N. Revaprasadu, "An improved N,Ndimethylformamide and polyvinylpyrrolidone approach for the synthesis of silver nano wires," Journal of Alloys and Compounds, vol. 469, pp. 519-522, 2009.

[15] M. Szymańska-Chargot, A. Gruszecka, A. Smolira et al., "Formation of nanoparticles and nanorods via UV irradiation of $\mathrm{AgNO}_{3}$ solutions," Journal of Alloys and Compounds, vol. 486, no. 1-2, pp. 66-69, 2009.

[16] S. Chen and D. L. Carroll, "Synthesis and characterization of truncated triangular silver nanoplates," Nano Letters, vol. 2, no. 9, pp. 1003-1007, 2002.

[17] S. Chen, Z. Fan, and D. L. Carroll, "Silver nanodisks: synthesis, characterization, and self-assembly ," Journal of Physical Chemistry B, vol. 106, no. 42, pp. 10777-10781, 2002.

[18] A. P. Kulkarni, K. M. Noone, K. Munechika, S. R. Guyer, and D. S. Ginger, "Plasmon-enhanced charge carrier generation in organic photovoltaic films using silver nanoprisms," Nano Letters, vol. 10, no. 4, pp. 1501-1505, 2010.

[19] R. Jin, Y. Cao, C. A. Mirkin, K. L. Kelly, G. C. Schatz, and J. G. Zheng, "Photoinduced conversion of silver nanospheres to nanoprisms," Science, vol. 294, no. 5548, pp. 1901-1903, 2001.
[20] J. An, B. Tang, X. Ning et al., "Photoinduced shape evolution: from triangular to hexagonal silver nanoplates," Journal of Physical Chemistry C, vol. 111, no. 49, pp. 18055-18059, 2007.

[21] Q. Zhang, J. Ge, T. Pham et al., "Reconstruction of silver nanoplates by UV irradiation: tailored optical properties and enhanced stability," Angewandte Chemie, vol. 48, no. 19, pp. 3516-3519, 2009.

[22] M. Tsuji, M. Hashimoto, Y. Nishizawa, M. Kubokawa, and T. Tsuji, "Microwave-assisted synthesis of metallic nanostructures in solution," Chemistry, vol. 11, no. 2, pp. 440-452, 2005.

[23] Y. J. Xiong, A. R. Siekkinen, J. G. Wang, Y. Yin, M. J. Kim, and Y. Xia, "Synthesis of silver nanoplates at high yields by slowing down the polyol reduction of silver nitrate with polyacrylamide," Journal of Materials Chemistry, vol. 17, no. 25, pp. 2600-2602, 2007.

[24] C. H. Kuo, T. F. Chiang, L. J. Chen, and M. H. Huang, "Synthesis of highly faceted pentagonal- And hexagonal-shaped gold nanoparticles with controlled sizes by sodium dodecyl sulfate," Langmuir, vol. 20, no. 18, pp. 7820-7824, 2004.

[25] N. R. Jana, L. Gearheart, and C. J. Murphy, "Wet chemical synthesis of silver nanorods and nanowires of controllable aspect ratio," Chemical Communications, vol. 7, pp. 617-618, 2001.

[26] C. A. I. Xionghui and Z. Aixia, "Preparation of microsized silver crystals with different morphologies by a wet-chemical method," Rare Metals, vol. 29, p. 407, 2010.

[27] YU. C. Lu and K. S. Chou, "A simple and effective route for the synthesis of nano silver colloidal dispersions," Journal of the Chinese Institute of Chemical Engineers, vol. 39, no. 6, pp. 673-678, 2008.

[28] G. Caputo and E. Reverchon, "Use of urea as habit modifier in the supercritical antisolvent micronization of sulfathiazole," Industrial and Engineering Chemistry Research, vol. 46, no. 12, pp. 4265-4272, 2007.

[29] R. Fuchs, "Theory of the optical properties of ionic crystal cubes," Physical Review B, vol. 11, no. 4, pp. 1732-1740, 1975.

[30] S. Chen and D. L. Carroll, "Silver nanoplates: size control in two dimensions and formation mechanisms," Journal of Physical Chemistry B, vol. 108, no. 18, pp. 5500-5506, 2004.

[31] J. Feng and H. C. Zeng, "Size-controlled growth of $\mathrm{Co}_{3} \mathrm{O}_{4}$ nanocubes," Chemistry of Materials, vol. 15, no. 14, pp. 28292835, 2003.

[32] Y. Xiong and Y. Xia, "Shape-controlled synthesis of metal nanostructures: the case of palladium," Advanced Materials, vol. 19, no. 20, pp. 3385-3391, 2007.

[33] C. J. Murphy and N. R. Jana, "Controlling the aspect ratio of inorganic nanorods and nanowires," Advanced Materials, vol. 14, no. 1, pp. 80-82, 2002.

[34] P. Jiang, J. J. Zhou, R. Li et al., "PVP-capped twinned gold plates from nanometer to micrometer," Journal of Nanoparticle Research, vol. 8, no. 6, pp. 927-934, 2006.

[35] Y. Yang, S. Matsubara, L. Xiong, T. Hayakawa, and M. Nogami, "Solvothermal synthesis of multiple shapes of silver nanoparticles and their SERS properties," Journal of Physical Chemistry C, vol. 111, no. 26, pp. 9095-9104, 2007. 

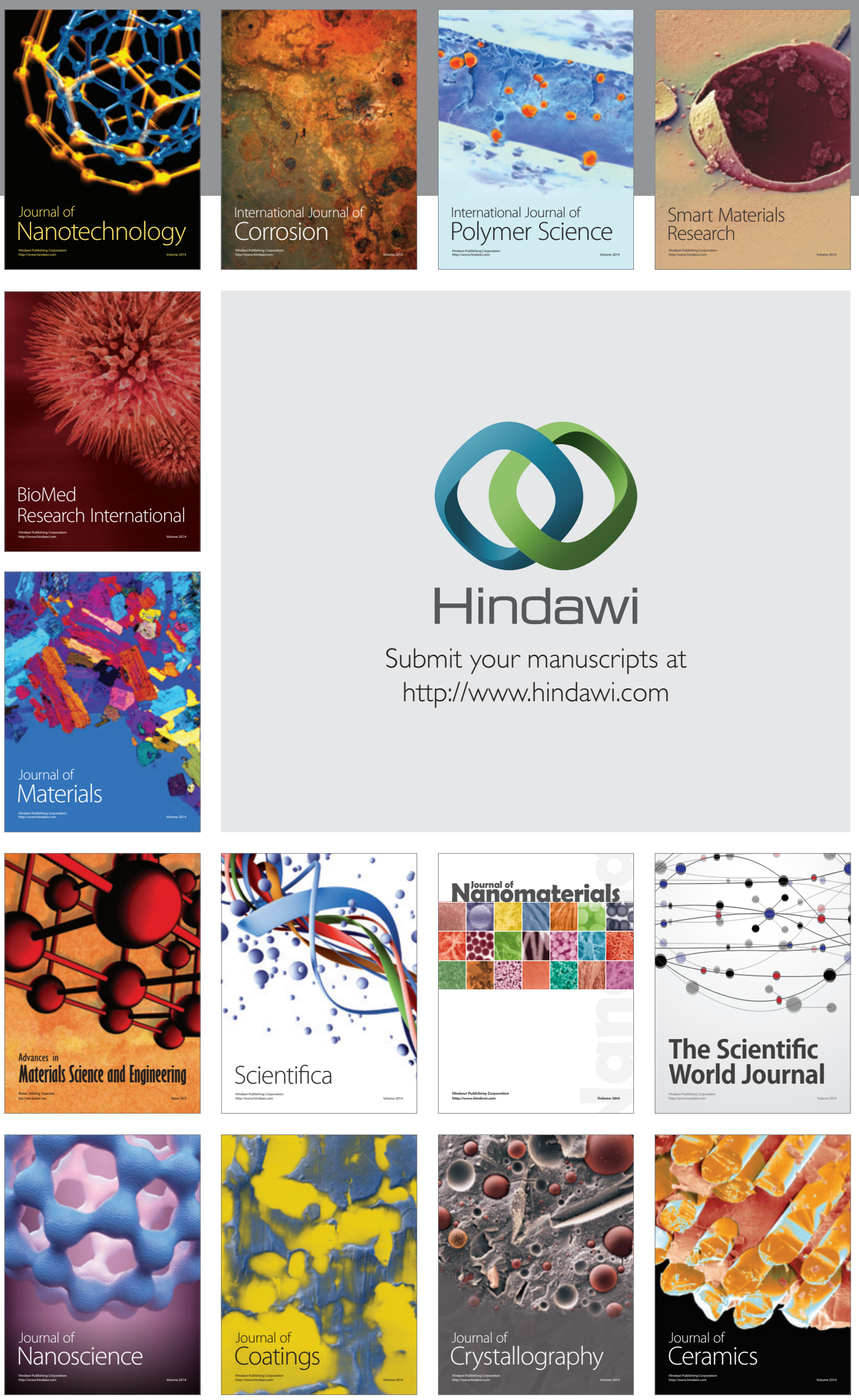

The Scientific World Journal

Submit your manuscripts at

http://www.hindawi.com

\section{World Journal}

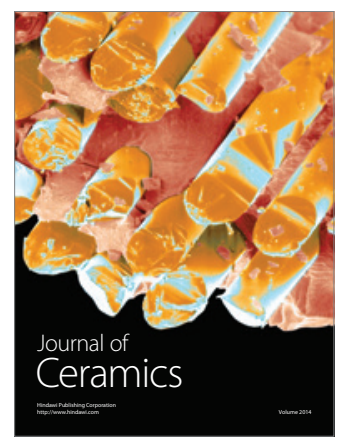

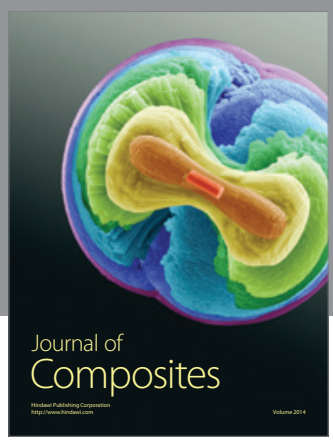
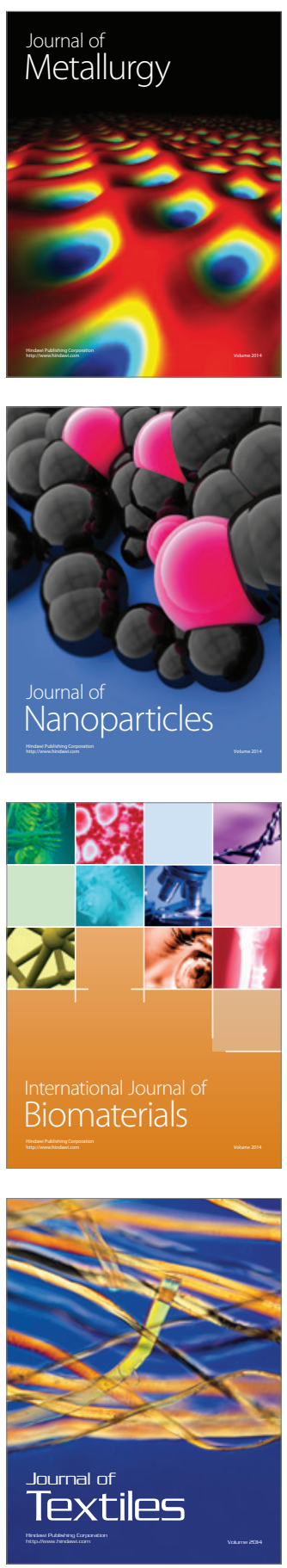